\title{
ADDENDUM
}

\section{Robust Arctic sea-ice influence on the frequent Eurasian cold winters in past decades}

Masato Mori, Masahiro Watanabe, Hideo Shiogama, Jun Inoue and Masahide Kimoto

Nature Geoscience 7, 869-873 (2014); published online 26 October 2014; corrected after print 16 January 2015.

We would like to draw attention to the colour scales in Fig. 1. Specifically, we used different colour scales for the panels showing reanalysis data $(\mathbf{a}, \mathbf{c})$ and those showing model ensembles $(\mathbf{b}, \mathbf{d})$. In $\mathbf{a}$ and $\mathbf{b}$ only the negative parts of the colour scale (blue shades) differ, by a factor of four. In $\mathbf{c}$ and $\mathbf{d}$ the entire colour scale is different.

This does not imply that the model underestimates observed atmospheric responses to the Arctic sea-ice reduction: owing to small sample size, a and c contain a considerable signal associated with the Arctic Oscillation, which accounts for about half of negative temperature anomalies over central Eurasia (Supplementary Infomation 1, Supplementary Fig. 5); the Arctic Oscillation signal is largely eliminated from the model ensemble mean (Supplementary Fig. 6). In contrast, central Eurasian temperature anomalies associated with the WACE pattern-the robust circulation response to sea-ice loss-were in good agreement between observations (-0.8 K) and model ensemble mean $(-0.6 \mathrm{~K})$ (Supplementary Figs 5,6). 